\title{
Effect of a wall on three-dimensionally unstable trailing vortices from a delta wing
}

\author{
Sarah E. Morris $\oplus^{*}$ and C. H. K. Williamson \\ Sibley School of Mechanical and Aerospace Engineering, Cornell University, \\ Ithaca, New York 14853, USA
}

(Received 22 January 2019; published 24 October 2019)

\begin{abstract}
This paper is associated with a poster winner of a 2018 APS/DFD Milton van Dyke Award for work presented at the DFD Gallery of Fluid Motion. The original poster is available online at the Gallery of Fluid Motion, https://doi.org/10.1103/APS.DFD.2018. GFM.P0020.
\end{abstract}

DOI: 10.1103/PhysRevFluids.4.100510

Understanding vortex-wall interactions has applications in fundamental turbulence, as well as being of interest in the context of airplane trailing vortices. Although seemingly simple, counterrotating vortex pairs can produce complex three-dimensional dynamics. In this work, we examine the development of a spatially evolving vortex pair with streamwise (axial) flow in the vortex core. The vortex pair is generated by towing a $75^{\circ}$ leading-edge sweep-angle delta wing in an $X Y$-Towing Tank, at a $15^{\circ}$ angle of attack. The facility also includes a removable, transparent acrylic ground plane for experiments in ground effect.

A key feature of this experiment is the ability to selectively visualize different features of the flow via laser-induced fluorescence. The flow is illuminated using an argon-ion laser. In Fig. 1, the vortex

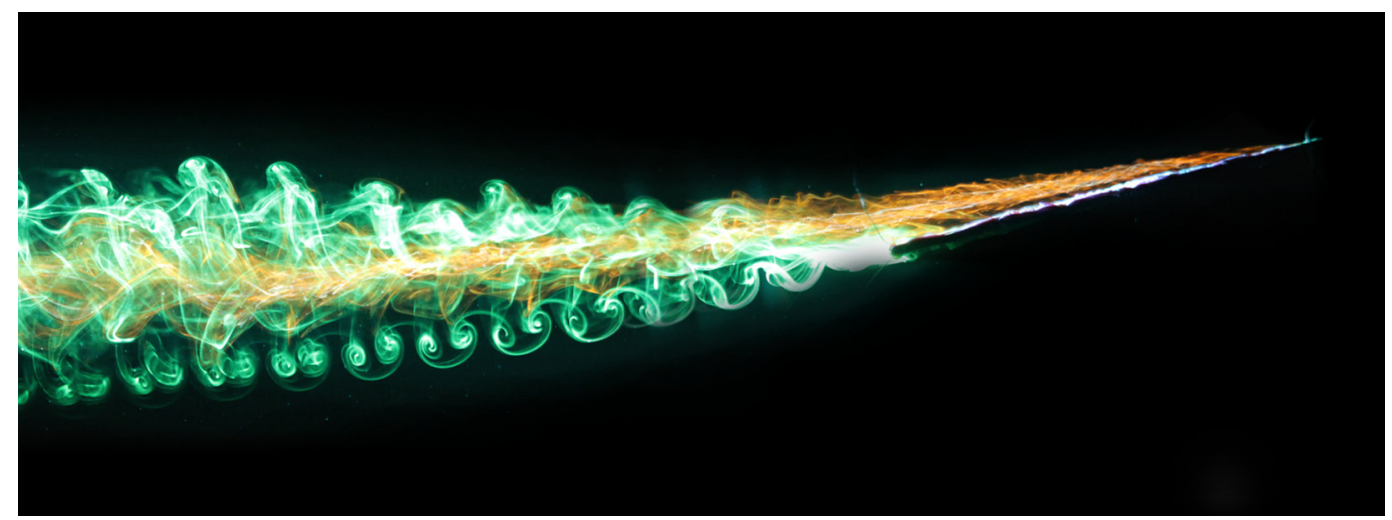

FIG. 1. A delta wing is towed in an $X Y$-Towing Tank, generating a counter-rotating vortex pair. The vortex cores are marked with rhodamine dye, and small-scale instabilities are made visible with fluorescein dye.

\footnotetext{
*sem323@ cornell.edu
}

Published by the American Physical Society under the terms of the Creative Commons Attribution 4.0 International license. Further distribution of this work must maintain attribution to the author(s) and the published article's title, journal citation, and DOI. 

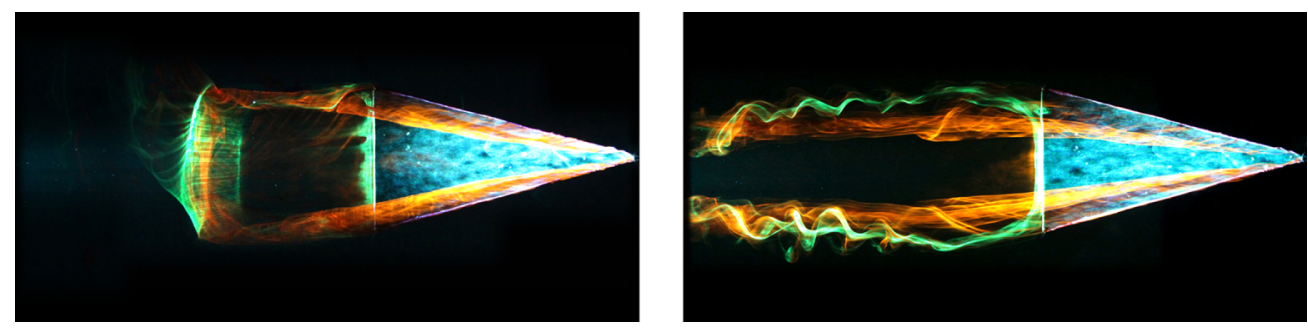

FIG. 2. Development of the vortex pair from rest behind a delta wing.

core is made visible by directly painting the apex of the delta wing with rhodamine dye. Fluorescein dye applied to the trailing edge of the delta wing marks small-scale instabilities wrapping around the vortex core, which are of the same wavelength as the von Kármán vortex street "braid wake" [1] behind the trailing edge.

Figure 2 shows a plan view of the starting vortex behind the delta wing. As the delta wing reaches its steady-state velocity, the fluorescein dye originating from the trailing edge wraps around the vortex core. The vortex core is made visible by rhodamine dye, using the same dye application technique as in Fig. 1.

When the vortex pair impinges on a wall, the boundary layer that forms on the surface between the vortices and the wall separates, generating secondary vorticity and causing the primary vortex pair to "rebound." Vorticity isocontours generated from particle image velocimetry (PIV) data show the spatial generation of secondary vorticity in Fig. 3. The initial generation of secondary vorticity is observed at the surface of the wall; in the far wake, the secondary vortex rolls up around the primary vortices.

Figure 4 shows the vortex pair in ground effect in the far wake of the delta wing. Fluorescein dye from the trailing edge has wrapped around the vortex core (rhodamine dye) and no longer has a defined structure. The ground plane is illuminated in blue by the argon-ion laser. We measure the axial flow velocity profile $v_{z}(r)$ in the vortex core more than 20 chord-lengths downstream of the delta wing using an oblique-section PIV technique as explored by Miller and Williamson [2]. For our delta wing of span $=6 \mathrm{~cm}$, this equates to data over $2 \mathrm{~m}$ downstream of the delta wing. The axial velocity profile in the vortex core is Gaussian, the shape of which is a function of time (distance downstream). A wakelike velocity deficit (flow upstream) is observed in the far wake of the delta wing, both in and out of ground effect.
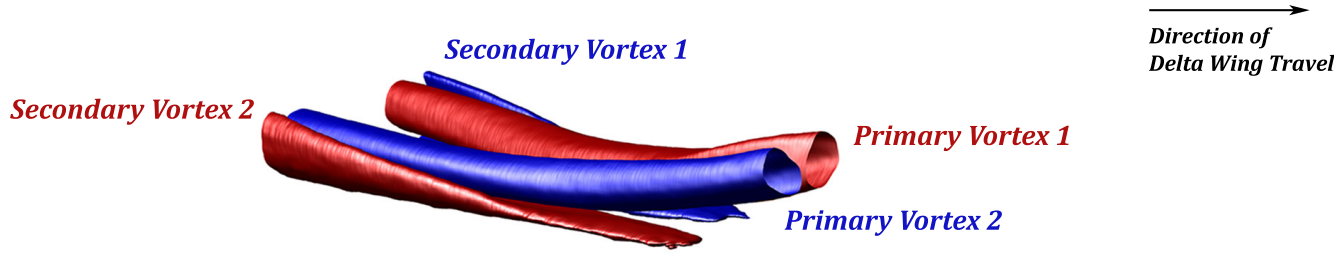

FIG. 3. Vorticity isocontours generated from PIV data. A primary vortex (e.g., Primary Vortex 1) with counterclockwise signed vorticity will generate a secondary vortex (e.g., Secondary Vortex 1) with clockwise rotation, and vice versa. 


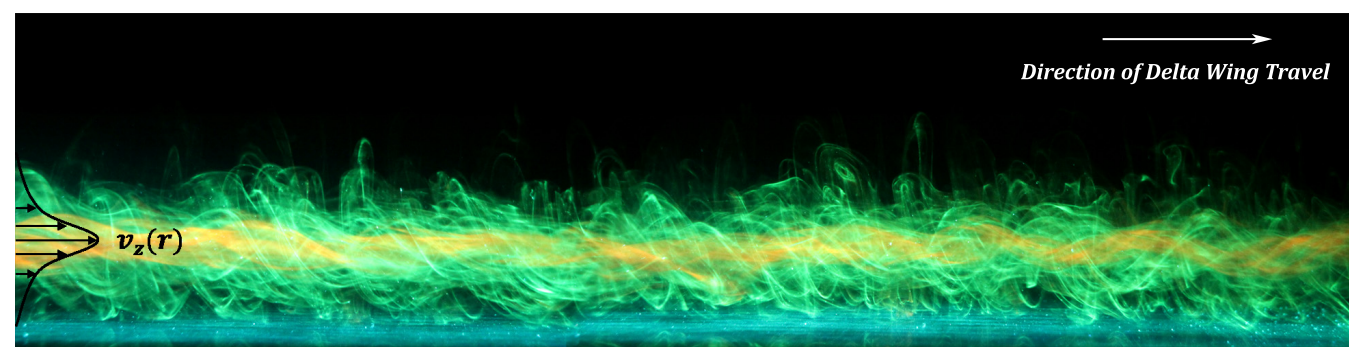

FIG. 4. Flow visualization of a vortex pair in ground effect, showing the direction and distribution of the axial velocity profile $v_{z}(r)$ in the vortex core(s).

[1] C. H. K. Williamson, T. Leweke, and G. D. Miller, Fundamental instabilities in spatially-developing wing wakes and temporally-developing vortex pairs, in Turbulence Structure and Vortex Dynamics, edited by J. C. R. Hunt and J. C. Vassilicos (Cambridge University Press, Cambridge, 2000), pp. 83-103.

[2] G. D. Miller and C. H. K. Williamson, Turbulence in the wake of a delta wing, in Proceedings of the Sixth European Turbulence Conference (Springer, Berlin, 1996). 